\title{
Real-time optical mapping of the dynamics of nonthermal electrons in thin gold films
}

\author{
G. Della Valle, ${ }^{1}$ M. Conforti, ${ }^{2}$ S. Longhi,${ }^{1}$ G. Cerullo, ${ }^{1}$ and D. Brida ${ }^{1, *}$ \\ ${ }^{1}$ Dipartimento di Fisica and Istituto di Fotonica e Nanotecnologie-Consiglio Nazionale delle Ricerche, Politecnico di Milano, \\ Piazza L. da Vinci 32, I-20133 Milan, Italy \\ ${ }^{2}$ Consorzio Nazionale Interuniversitario per le Scienze Fisiche della Materia, Dipartimento di Ingegneria dell'Informazione, \\ Università di Brescia, Via Branze 38, I-25123 Brescia, Italy \\ (Received 23 May 2012; revised manuscript received 2 October 2012; published 22 October 2012)
}

\begin{abstract}
We investigate the nonlinear optical response of thin gold films with an unprecedented combination of high temporal resolution $(\sim 15 \mathrm{fs})$ and broad spectral coverage. We quantitatively model the data without free parameters using an extended version of the two-temperature model. Our combined experimental and theoretical approach allows tracking the evolution from a purely nonthermal electron distribution towards a fully thermalized one. These results pose the basis for better understanding of light-matter interaction in metals on the ultrafast time scale.
\end{abstract}

DOI: 10.1103/PhysRevB.86.155139

PACS number(s): 78.66.Bz, 78.40.Kc, 78.47.jb

\section{INTRODUCTION}

With the recent advent of nanoscience, noble metals have experienced an authentic renaissance. The unprecedented capability of controlling light-matter interaction at the nanoscale has led to the demonstration of fascinating phenomena in plasmonic systems and metamaterials, such as subwavelength propagation and focusing of light (see Ref. 1 and references therein) as well as the observation of giant noninstantaneous optical nonlinearities. ${ }^{2-4}$ Very recently, a new frontier in nano-optics has been disclosed aimed at the exploration of nonlinear optical processes taking place on the few-fs timescale, with major impact on both basic and applied research. After the pioneering demonstration of ultrafast coherent control of photoemission from metal nanostructures, ${ }^{5}$ several groundbreaking experiments have been reported in the last years, including: efficient nonlinear light emission of single gold optical antennas driven by few-cycle near-infrared pulses; ${ }^{6,7}$ few-fs resolution investigation of the dephasing of plasmonic oscillation in a single metallic nanostructure ${ }^{8}$ and corrugated metal surfaces by coherent two-dimensional nanoscopy; ${ }^{9}$ and ultrafast plasmon propagation in silver nanowires. ${ }^{10}$ Furthermore, the combination of subwavelength localization, leading to intensity enhancement of the local fields, and the intrinsic giant nonlinearity of the metal offers the rather unique possibility to explore strong-field physics in the solid state ${ }^{11}$ and eventually merge attosecond science with plasmonics. ${ }^{12}$ On the other hand, manipulation and control of electron dynamics on the femtosecond time scale in metallic nanostructures is also of major importance in the development of active nanodevices, for applications to all-optical switching. ${ }^{13,14}$ This large body of works clearly highlights the importance of understanding, and experimentally accessing, the primary ultrafast electron relaxation processes in metals occurring on a few-femtosecond timescale.

The response time of metal-based nanostructures is ultimately limited by the ultrafast electronic dynamics. In this respect, the thin metal film, representing the simplest kind of nanosized gold, plays a key role. Several papers have been so far devoted to the study of ultrafast optical dynamics of thin gold and silver films. These studies have indicated that the nonlinear optical response is dominated by electron-electron interactions (EEIs) and electron-phonon interactions (EPIs). ${ }^{15-18}$ Impulsive optical excitation creates an out-of-equilibrium, nonthermal electron distribution, which then evolves on the tens of fs timescale through EEI and EPI into a thermalized distribution; subsequent equilibration with the cold lattice occurs mainly through EPI on the slower, ps timescale. While EPI has been experimentally characterized in detail on the long timescale and satisfactorily described by the two-temperature model (TTM), the early electron thermalization, mainly driven by EEI, is more challenging to study, due to its ultrafast nature and complex nonequilibrium interactions. Also, the role played by pump photon energy has not been investigated.

In this paper we perform an extensive experimental and theoretical investigation of the nonlinear optical response of thin gold films with a combination of high temporal resolution ( $\sim 15 \mathrm{fs}$ ) and broad spectral coverage. Our combined approach allows tracking the evolution from a purely nonthermal electron distribution towards a fully thermalized one also in dependence of the pump photon energy. These findings pose the basis for better understanding of light-matter interaction in metals on the ultrafast time scale that is exploited in plasmonics and high-field physics.

\section{EXPERIMENTS}

When the photon energy $h v_{p}$ is lower than the interband optical transition edge $(\sim 1.8 \mathrm{eV}$ in gold $)$ the interaction of intense (i.e., pump) light pulses with noble metal structures gives rise to intraband absorption processes [Fig. 1(a)]. ${ }^{15,16}$ Correspondingly, the electron occupation probability is strongly perturbed from the thermal equilibrium Fermi-Dirac function $f_{0}(E)$ by an amount that is commonly assumed to be a double steplike function (see, e.g., Ref. 15) extending from $-h v_{p}$ to $+h v_{p}$ around Fermi energy $E_{F}$ [see dotted red line in Fig. 1(b)] This strongly out-of-equilibrium distribution relaxes on the ultrafast time scale through the EEI and EPI processes.

The experimental setup used for high time resolution broadband pump-probe spectroscopy is described in detail in Refs. 19-21. Both pump and probe pulses are generated by noncollinear optical parametric amplifiers at $1 \mathrm{kHz}$. Pump 

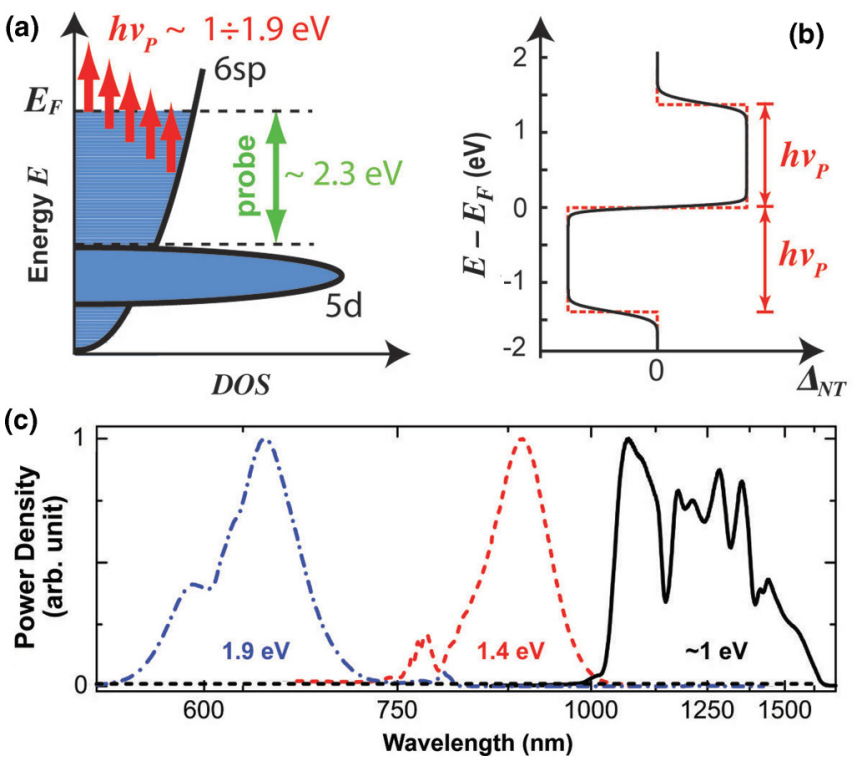

FIG. 1. (Color online) (a) Drawing of gold density of states showing intraband pump absorption (red arrows) and interband probe optical transitions (green arrow). (b) Pump-induced variation of the electron energy occupation probability for the broadband pump pulses considered in our experiments (solid black line) as compared to the steplike idealization assumed in Ref. 24 (dotted red line). (c) Power spectral density of the pump pulses employed in the experiment.

pulses have 10-12 fs duration and peak photon energy tunable in the range 1-1.9 eV [Fig. 1(c)], while the broadband sub-10-fs probe pulses have a spectrum ranging from 2.1 to $2.55 \mathrm{eV}$ (i.e., 490-580 nm), thus covering the frequency range of gold interband transitions. The probe pulse is dispersed on a spectrometer, allowing acquisition of two-dimensional differential transmission $(\Delta T / T)$ maps as a function of pumpprobe delay $t$ and probe wavelength $\lambda$. This nondegenerate pump-probe configuration eliminates coherent interference artifacts and allows independent and broadband tunability of the pump and of the probe, as well as parallel acquisition of transients at different probe photon energies, leading to $\Delta T / T$ maps, which allow an accurate description of the electron relaxation process. Experiments are performed on a 30-nm thin gold film evaporated on silica glass substrate (refractive index $n_{S}=1.42$ ).

The experimental $\Delta T / T$ map recorded for pump photon energy of $1.4 \mathrm{eV}$ is reported in Fig. 2(a). Immediately after the pump pulse, the $\Delta T / T$ signal is negative at all probe photon energies, with a rather flat frequency dependence that is consistent with the impulsive buildup of a broadly dispersed nonthermal distribution [cf. Fig. 1(b)]. Within 200 fs, the negative band grows and narrows considerably, peaking at around $503 \mathrm{~nm}(2.465 \mathrm{eV})$, close to the $d$ band to Fermi level energy $\operatorname{gap}^{22}(\sim 2.3 \mathrm{eV})$. Simultaneously, the $\Delta T / T$ signal turns positive in the red wing of the transient spectrum. The isosbestic line (i.e., locus of zero in the $\Delta T / T$ map) [black in Fig. 2(a)] then shifts to the blue with time [see Fig. 2(c)]. This complex spectral evolution reflects the combination of EEI and EPI processes during electron thermalization, which we can follow in real time thanks to the combination of high temporal resolution and broad spectral coverage.

\section{THEORETICAL MODEL}

Transient absorption measurements in thin gold films have been interpreted according to several theoretical approaches of different complexity, ranging from the simplest semiclassical TTM, only accounting for the slower EPI process (see, e.g., Ref. 23), to the more sophisticated ab initio nonthermal electron model (NEM) involving brute-force numerical solution of Boltzmann equations. ${ }^{15,16}$ Though it has been demonstrated that only the NEM is capable of capturing the important role played by the ultrafast EEI (responsible to the doubling of the electron energy relaxation time observed in the experiments as compared to the TTM prediction), ${ }^{16}$ its numerical complexity prevents exploitation of this approach for further analytical developments. On the other hand, the ordinary TTM is a rather simple and appealing model; however, it fails to predict some basic physical features of the ultrafast relaxation dynamics of metallic systems.

We have modeled our experimental results adopting an extended version of the TTM, which has been recently proposed with the aim of grasping the essentials of the EEI mechanisms detailed by Boltzmann equations, without abandoning the simple formalism of a two-coupled-equations model. The extended TTM (ETTM) is described by the following coupled equations for the temperature $T_{e}$ of thermalized electrons and the lattice temperature $T_{l}: 24$

$$
\begin{aligned}
C_{e} \frac{d T_{e}}{d t} & =-g\left(T_{e}-T_{l}\right)+\int P_{a}\left(t^{\prime}\right) H_{e e}\left(t-t^{\prime}\right) d t^{\prime} \\
C_{l} \frac{d T_{l}}{d t} & =g\left(T_{e}-T_{l}\right)+\int P_{a}\left(t^{\prime}\right) H_{e p}\left(t-t^{\prime}\right) d t^{\prime},
\end{aligned}
$$

where $C_{e}=\gamma T_{e}$ and $C_{l}$ are the electronic and lattice volumespecific heat capacities, $g$ is the electron-phonon coupling constant, $P_{a}(t)$ is the pump pulse power absorbed in the unit volume of the metal, and $H_{e e}(t)$ and $H_{e p}(t)$ account for the energy transfer from nonthermalized electrons (generated by pump excitation) to thermalized electrons and to the lattice, respectively. Their explicit form is given in the Appendix.

The ETTM allows one to consistently calculate the temporal evolution of $T_{e}$, and thus the corresponding contribution to the variation of electron distribution accounting for thermalized $\Delta f_{T}$ and nonthermalized $\Delta f_{N T}$ electrons. The former contribution is given by $\Delta f_{T}(E, t)=f_{0}\left[E, T_{e}(t)\right]-f_{0}\left(E, T_{0}\right), T_{0}$ being the room temperature, whereas the latter contribution is computed, similarly to Ref. 24 , as:

$$
\Delta f_{N T}(E, t)=1 / A \times \Delta_{N T}(E) \int_{-\infty}^{t} P_{a}\left(t^{\prime}\right) e^{-\left(t-t^{\prime}\right) / \tau(E)} d t^{\prime},
$$

where the explicit expressions of $\tau(E), \Delta_{N T}(E)$ and normalization constant $A$ are given in the Appendix. Here we just notice that $\Delta_{N T}(E)$ is determined from the measured power spectral density of the pump pulse normalized to the pump pulse energy $S_{p}(h v)$ [Fig. 1(c)] to take into account the broad excitation bandwidth, thus generalizing the previous approach reported in Ref. 24 [cf. Fig. 1(b)]. The total variation of the electron energy distribution due to thermal and nonthermal electrons results in a reduction (increase) of the occupation probability of the electron states below (above) $E_{F}$. As a consequence, a modulation of interband transition 

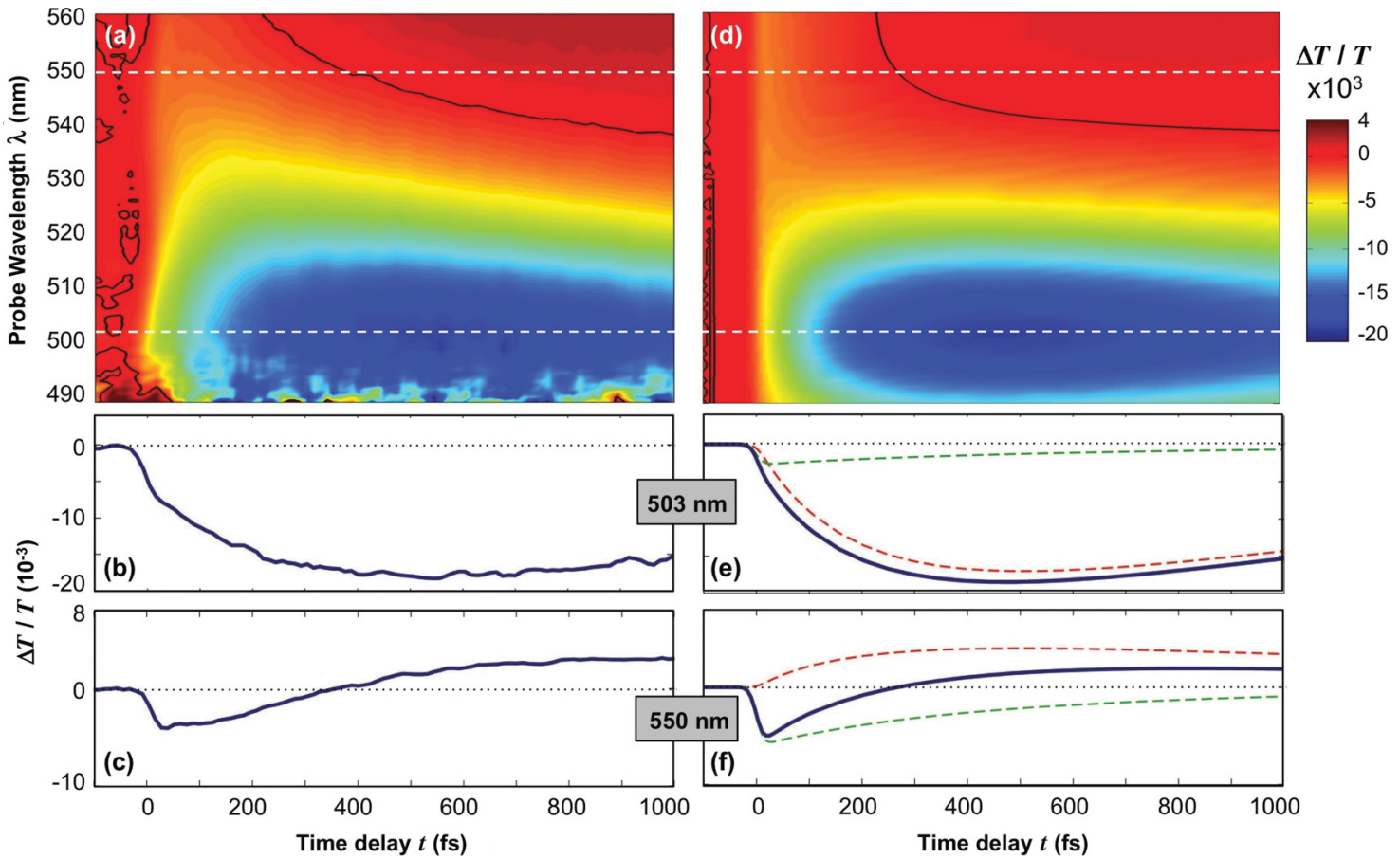

FIG. 2. (Color online) $\Delta T / T$ maps and representative cross sections obtained from experimental measurements (left panels) and numerical simulations (right panels), for the pump pulse at $1.4 \mathrm{eV}$ reported in Fig. 1(c), and incident pump fluence $F=430 \mu \mathrm{J} / \mathrm{cm}^{2}$. Dashed lines in right panels represent the contribution to the total $\Delta T / T$ signal due to (green) nonthermalized electrons and (red) thermalized electrons. For simulations, $P_{a}(t)=F_{a} / d \times p(t)$, being $F_{a}=\left(1-R_{p}-T_{p}\right) F$ the absorbed fluence computed from the reflectance $R_{p}$ and transmittance $T_{p}$ of the thin film at the pump peak wavelength, and $p(t)=\exp \left(-2 t^{2} / t_{p}^{2}\right) /\left(\sqrt{\pi / 2} t_{p}\right)$ with $t_{p}=20 \mathrm{fs}$, corresponding to the time duration of the measured pump-pulse cross correlation.

probability is induced, with increased (decreased) absorption for transitions involving final states below (above) $E_{F}$. In the constant matrix element approximation, the variation of the imaginary part of the interband dielectric function of gold at the probe wavelength $\lambda$ due to the pump-induced perturbation of thermal (T) and nonthermal (NT) electron energy distributions can be computed as follows: ${ }^{25}$

$$
\Delta \varepsilon_{T(N T)}^{\prime \prime}(\lambda, t)=\frac{e^{2} \lambda^{2}}{3 m^{2} c^{2}}\left|P_{L}\right|^{2} \Delta J_{T(N T)}(\lambda, t),
$$

where $m$ is the free electron mass, $P_{L}$ is the electric-dipole matrix element, and $\Delta J_{T(N T)}$ is the pump-induced variation of the joint density of states (JDOS) for $d$-band to conduction band transitions near $L$ point in the irreducible zone of the Brillouin cell. Such variation can be computed as ${ }^{25}$

$$
\Delta J_{T(N T)}(\lambda, t)=\int_{E^{\prime}}^{E^{\prime \prime}} D(E, \lambda) \Delta P_{T(N T)}(E, t) d E,
$$

where $D(E, \lambda)$ is the energy distribution of the joint density of states (EDJDOS) of the considered optical transition (with respect to the energy of final state $E$ ), and $\Delta P_{T(N T)}=$ $-\Delta f_{T(N T)}$ is the variation of final state occupation probability for thermalized (nonthermalized) electrons. The EDJDOS was numerically computed under parabolic band approximation following the approach described in Ref. 25, taking effective masses, energy gaps, dipole matrix element, and integration limits $E^{\prime}$ and $E^{\prime \prime}$ as reported in Ref. 22. The variation of the real part of the interband dielectric function $\Delta \varepsilon_{T(N T)}^{\prime}(\lambda, t)$ is determined by Kramers-Krönig analysis of $\Delta \varepsilon_{T(N T)}^{\prime \prime}(\lambda, t)$.
Additional contributions to the change in the dielectric constant, given by intraband transitions that depend on lattice temperature, are neglected since we are interested in the very initial (sub-ps) part of the dynamics, where the lattice temperature is almost constant and equal to room temperature. With the time-dependent complex dielectric function of the metal provided by Eqs. (1)-(4) at hand, we numerically modeled the $\Delta T / T(\lambda, t)$ signal of the thin gold film according to standard thin film formulas. ${ }^{26}$ Parameters for the ETTM were taken from literature $17,24,27$ as $\gamma=68 \mathrm{~J} \mathrm{~m}^{-3} \mathrm{~K}^{-2}$, $C_{l}=2.5 \times 10^{6} \mathrm{~J} \mathrm{~m}^{-3} \mathrm{~K}^{-1}, g=2.2 \times 10^{16} \mathrm{~W} \mathrm{~m} \mathrm{~m}^{-3} \mathrm{~K}^{-1}$, $\tau_{f}=14 \mathrm{fs}, \tau_{0}=7 \mathrm{fs},{ }^{28} E_{F}=7.3 \mathrm{eV}$. Results are reported in Figs. 2(d)-2(f), and show a good quantitative agreement with the experimental data without any free parameters.

\section{DISCUSSION}

We found that the sign change, from negative to positive, observed in the $\Delta T / T$ dynamics for lower pump photon energies is precisely ascribable to the interplay between the two contributions arising from thermal and nonthermal electrons [Fig. 2(f)]. We also investigated the effect of pumping with different pump photon energies by exploiting the capability of our setup to achieve ultrabroadband and independent tuning of the pump and probe pulses. We found that $\Delta T / T$ maps measured at $h v_{p}=1 \mathrm{eV}$ and $h v_{p}=1.9 \mathrm{eV}$ exhibit the same qualitative spectral features observed in the $\Delta T / T$ map of Fig. 2, attained at $h v_{p}=1.4 \mathrm{eV}$, and in particular the peak of the $\Delta T / T$ signal is in any case observed at $\lambda \simeq 503 \mathrm{~nm}$. 


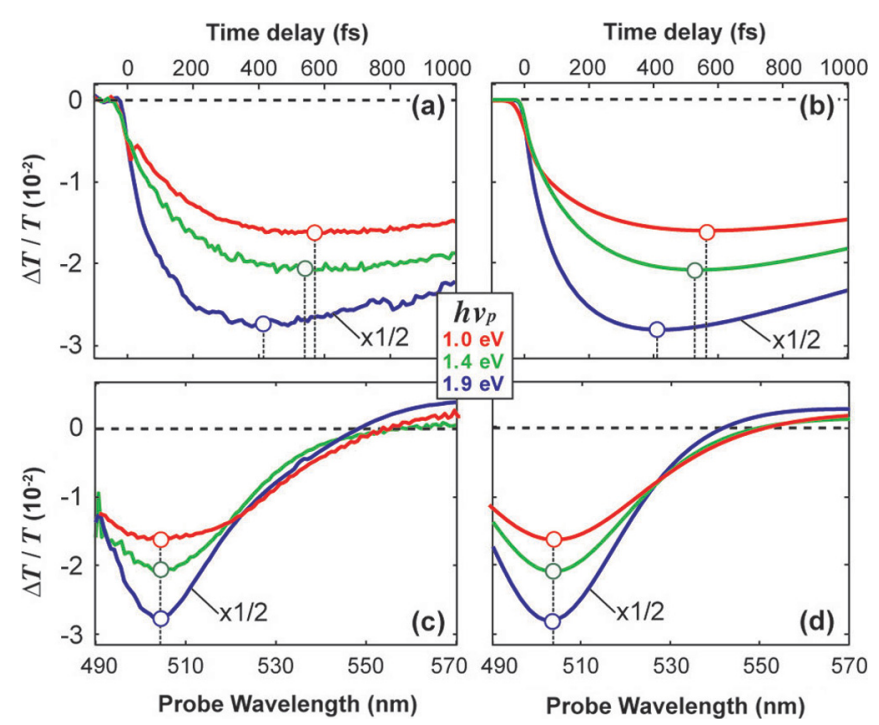

FIG. 3. (Color online) Effect of pump photon energy. (a) Experimental and (b) theoretical $\Delta T / T$ temporal dynamics at $\lambda=503 \mathrm{~nm}$. (c) Experimental and (d) theoretical $\Delta T / T$ spectra at the time delay of signal peak (i.e. $t=580,540$ and $410 \mathrm{fs}$, for $h v_{p}=1.0,1.4$, and $1.9 \mathrm{eV}$ respectively). Incident pump fluence as in Fig. 2.

On the other hand, we found that the temporal position of this peak is reached at different time delays, from $\sim 410 \mathrm{fs}$ to $\sim 580$ fs [Fig. 3(a)], thus indicating a dependence of the rise time of the delayed nonlinear response on the pump photon energy, as also confirmed by numerical simulations [Fig. 3(b)]; the variation on the thermalization dynamics is also reflected in the time-dependent frequency shift of the isosbestic point that results also in different spectral width of the peak at $503 \mathrm{~nm}$ probe wavelength [Figs. 3(c)-3(d)]. This unexpected behavior can be explained by the following argument. Considering the effect of the two contributions to $\Delta T / T$ arising from thermal and nonthermal electrons [cf. Figs. 2(e)-2(f)], it is evident that an acceleration of the nonlinear transients responsible to the formation of the signal peak is ascribable to an acceleration of the $\Delta T / T$ contribution coming from thermalized electrons, which in the perturbative regime of our experiment is proportional to $-\Delta T_{e}$. From Eq. (1), ideally assuming a $\delta$ function for the pump pulse [i.e., $p(t)=\delta(t)]$, the time derivative of the electron temperature right after pump absorption is approximately given by

$$
\left[\frac{d T_{e}}{d t}\right]_{t=0^{+}} \simeq \frac{F_{a}}{\gamma T_{0} d} \lim _{t \rightarrow 0^{+}} H_{e e}(t)=\frac{F_{a}\left(h v_{p}\right)^{2}}{2 \gamma T_{0} d E_{F}^{2} \tau_{0}},
$$

which scales with the square of pump photon energy.

This precisely indicates that by increasing pump photon energy an acceleration of the dynamics of thermal electrons takes place and is a consequence of the fact that electrons with higher excitation energy above the Fermi level possess a faster scattering rate, as predicted by the Fermi liquid theory. Thanks to the excellent agreement between model and experiments, we can infer how the actual electronic distribution evolves in time starting from the nonequilibrium distribution created by the pump pulse. Figure 4 shows the calculated variation of the electronic distribution (black solid line) at various time delays for $h v_{p}=1.4 \mathrm{eV}$. The thermalized (hot) electron population

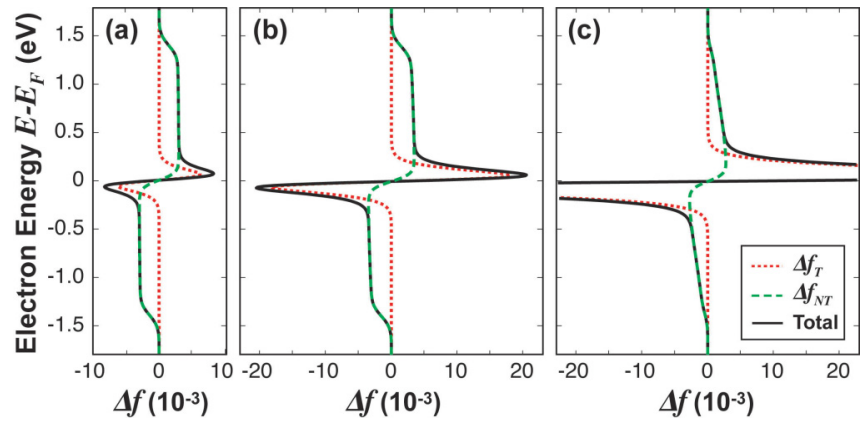

FIG. 4. (Color online) Theoretical reconstruction of the variation of electron energy distribution, according to the data of Fig. 2, at different time delays: (a) $10 \mathrm{fs}$; (b) $40 \mathrm{fs}$; (c) $300 \mathrm{fs}$.

(red dotted line) builds up within 500 fs and becomes the largely dominant contribution close to $E_{F}$. The nonthermal electron population (green dashed line), instead, is decreasing and its distribution becomes smoother, with maxima moving closer to $E_{F}$. It is worth noticing that its contribution is still significant after hundreds of fs thus explaining the continuous evolution of the transient optical spectra recorded in our experiments.

\section{CONCLUSION}

We have reported an experimental investigation of the ultrafast optical response of a thin gold film with an unprecedented combination of high temporal resolution and broad spectral coverage. Besides accurately mapping electron thermalization through the EEI process, we observe unexpected features, such as the dependence of the rise time of the delayed nonlinear response on the pump photon energy. An extended version of the TTM allowed us to provide accurate modeling of all the features exhibited by the thin gold film in the sub-ps time scale, without resorting to any fitting parameter and allowing us to infer the transient electronic distribution. It is worth saying that all the features investigated depend on the bulk properties of gold and on the hypothesis of having an optically thin gold material. Therefore our results are expected to provide physical insight into the ultrafast optical response exhibited by other optically thin gold structures as well as gold-based metamaterials and plasmonic media. Also, it is envisioned that the accuracy demonstrated by the holdable ETTM could stimulate further theoretical developments in the field, and in particular a more comprehensive formulation of the nonlinear susceptibility of noble metals, thus generalizing the approach recently introduced in Refs. 29 and 30 (only accounting for thermalized electrons), with potential impact on the next generation of ultrafast nonlinear devices based on noble metal nanostructures.

\section{ACKNOWLEDGMENTS}

We gratefully acknowledge E. Carpene and J. Demsar for the fruitful discussions. This work was supported by Fondazione Cariplo through the projects: "Engineering of optical nonlinearities in plasmonic metamaterials" (Grant No. 2010-0595) and "New Frontiers in Plasmonic Nanosensing" (Grant No. 2011-0338). 


\section{APPENDIX}

The extended TTM and nonthermalized electron distribution. The extended version of the TTM (ETTM) is defined by the two coupled equations (1) given in the text that govern the relaxation dynamics of the thermalized electron temperature $T_{e}$ and the lattice temperature $T_{l}$ after pump photoexcitation. In such equations, the energy transfer from non-thermalized electrons (generated by pump excitation) to thermalized electrons and to the lattice is described by the driving kernels $H_{e e}(t)$ and $H_{e p}(t)$, which can be derived from Boltzmann equations under relaxation-time approximation. ${ }^{16}$ Their explicit expressions is given by

$$
\begin{aligned}
& H_{e e}(t)=-\frac{e^{-\left(1 / \tau_{1}+1 / \tau_{e p}\right) t}}{t^{2}}\left[t+\tau_{1}\left(1-e^{t / \tau_{1}}\right)\right] H(t) \\
& H_{e p}(t)=-\frac{e^{-\left(1 / \tau_{1}+1 / \tau_{e p}\right) t}}{t \tau_{e p}}\left[\tau_{1}\left(1-e^{t / \tau_{1}}\right)\right] H(t)
\end{aligned}
$$

where $H(t)$ is the step (Heaviside) function, $\tau_{e p}$ is the electronphonon energy relaxation time, and the time constant $\tau_{1}$ is given by

$$
\tau_{1}=\frac{\tau_{0}}{\rho} .
$$

In the previous equation, $\tau_{0}$ is related to the electron-electron energy relaxation time $\tau_{e e}$ according to the Fermi liquid theory

$$
\tau_{e e}(E)=\frac{\tau_{0} E_{F}^{2}}{\left(E-E_{F}\right)^{2}},
$$

and $\rho=\left(h v_{p} / E_{F}\right)^{2}$. The electron-phonon energy relaxation time $\tau_{e p}$ is approximately given by ${ }^{16}$

$$
\tau_{e p}=\frac{\tau_{f} h v_{p}}{k_{B} \theta_{D}}
$$

where $\tau_{f}$ is the quasi-particle free flight time and $\theta_{D}$ is the Debye temperature of the metal.

The ETTM theory allows one to determine the temporal evolution of $T_{e}$ and thus the corresponding contribution $\Delta f_{T}$ to the variation of electron distribution $\Delta f$ accounting for thermalized electrons. Similarly to, ${ }^{16}$ the contribution $\Delta f_{N T}$ to $\Delta f$ due to non-thermalized electrons can be estimated according to Eq.(2) given in the text, where:

$$
\begin{gathered}
\tau(E)=\frac{\tau_{e p} \tau_{e e}(E)}{\tau_{e p}+\tau_{e e}(E)}, \\
\Delta_{N T}(E)=\int S_{p}(h \nu) \delta_{N T}(E, h \nu) d h \nu,
\end{gathered}
$$

and

$$
\begin{aligned}
\delta_{N T}(E, h v)= & f_{0}\left(E-E_{F}-h v\right)\left[1-f_{0}\left(E-E_{F}\right)\right] \\
& -f_{0}\left(E-E_{F}\right)\left[1-f_{0}\left(E-E_{F}+h v\right)\right] .
\end{aligned}
$$

Since in our model the pump absorption is solely ascribed to intra-band transitions leading to non thermalized electrons, the constant factor $A$ entering in Eq.(2) is determined by imposing energy conservation, yielding

$$
A=\int \Delta_{N T}(E) D O S(E) E d E,
$$

where $\operatorname{DOS}(E)$ is the energy density of states in gold. *daniele.brida@mail.polimi.it

${ }^{1}$ J. A. Schuller, E. S. Barnard, W. Cai, Y. C. Jun, J. S. White, and M. L. Brongersma, Nature Mater. 9, 193 (2010).

${ }^{2}$ R. S. Bennik, Y.-K. Yoon, R. W. Boyd, and J. E. Sipe, Opt. Lett. 24, 1416 (1999).

${ }^{3}$ G. Yang, D. Guan, W. Wang, W. Wu, and Z. Chen, Opt. Mater. 25, 439 (2004).

${ }^{4}$ A. Husakou and J. Herrmann, Phys. Rev. Lett. 99, 127402 (2007).

${ }^{5}$ M. Aeschlimann, M. Bauer, D. Bayer, T. Brixner, F. J. Garcia de Abajo, W. Pfeiffer, M. Rohmer, C. Spindler, and F. Steeb, Nature (London) 446, 301 (2007).

${ }^{6}$ T. Hanke, G. Krauss, D. Trautlein, B. Wild, R. Bratschitsch, and A. Leitenstorfer, Phys. Rev. Lett. 103, 257404 (2009).

${ }^{7}$ T. Hanke, J. Cesar, V. Knittel, A. Trgler, U. Hohenester, A. Leitenstorfer, and R. Bratschitsch, Nano Lett. 12, 992 (2012).

${ }^{8}$ A. Anderson, K. S. Deryckx, X. G. Xu, G. Steinmeyer, and M. B. Raschke, Nano Lett. 10, 2519 (2010).

${ }^{9}$ M. Aeschlimann, T. Brixner, A. Fischer, C. Kramer, P. Melchior, W. Pfeiffer, C. Schneider, C. Struber, P. Tuchscherer, and D. V. Voronine, Science 333, 1723 (2011).

${ }^{10}$ C. Rewitz, T. Keitzl, P. Tuchscherer, J.-S. Huang, P. Geisler, G. Razinskas, B. Hecht, and T. Brixner, Nano Lett. 12, 45 (2012).

${ }^{11}$ G. Herink, D. R. Solli, M. Gulde, and C. Ropers, Nature (London) 483, 190 (2012)
${ }^{12}$ M. Kruger, M. Schenk, and P. Hommelhoff, Nature (London) 475, 78 (2011).

${ }^{13}$ K. F. MacDonald, Z. L. Samson, M. I. Stockman, and N. I. Zheludev, Nature Photon. 3, 55 (2009).

${ }^{14}$ G. A. Wurtz, R. Pollard, W. Hendren, G. P. Wiederrecht, D. J. Gosztola, V. A. Podolskiy, and A. V. Zayats, Nature Nanotech. 6, 107 (2011).

${ }^{15}$ C.-K. Sun, F. Vallée, L. H. Acioli, E. P. Ippen, and J. G. Fujimoto, Phys. Rev. B 50, 15337 (1994).

${ }^{16}$ R. H. M. Groeneveld, R. Sprik, and A. Lagendijk, Phys. Rev. B 51, 11433 (1995).

${ }^{17}$ J. Hohlfeld, S.-S. Wellershoffm, J. Gudde, U. Conrad, V. Janke, and E. Matthias, Chem. Phys. 251, 237 (2000).

${ }^{18}$ N. Del Fatti, C. Voisin, M. Achermann, S. Tzortzakis, D. Christofilos, and F. Vallee, Phys. Rev. B 61, 16956 (2000).

${ }^{19}$ D. Brida, C. Manzoni, G. Cirmi, M. Marangoni, S. Bonora, P. Villoresi, S. De Silvestri, and G. Cerullo, J. Opt. 12, 013001 (2010).

${ }^{20}$ D. Brida, C. Manzoni, G. Cirmi, D. Polli, and G. Cerullo, IEEE J. Sel. Top. Quantum Electron. 18, 329 (2012).

${ }^{21}$ D. Brida, S. Bonora, C. Manzoni, M. Marangoni, P. Villoresi, S. De Silvestri, and G. Cerullo, Opt. Express 17, 12510 (2009).

${ }^{22}$ M. Guerrisi, R. Rosei, and P. Winsemius, Phys. Rev. B 12, 557 (1975).

${ }^{23}$ S. I. Anisimov, B. L. Kapeliovich, and T. L. Perelman, Sov. Phys. JETP 39, 375 (1974). 
${ }^{24}$ E. Carpene, Phys. Rev. B 74, 024301 (2006).

${ }^{25}$ R. Rosei, Phys. Rev. B 10, 474 (1974).

${ }^{26} \mathrm{~F}$. Abeles, in Advanced Optical Techniques, edited by Van Heel (North-Holland, Amsterdam, 1967), Chap. 5, p. 144.

${ }^{27}$ S. Link, C. Burda, M. B. Mohamed, B. Nikoobakht, and M. A. El-Sayed, Phys. Rev. B 61, 6086 (2000).
${ }^{28}$ W. S. Fann, R. Storz, H. W. K. Tom, and J. Bokor, Phys. Rev. B 46, 13592 (1992).

${ }^{29}$ H. Baida, D. Mongin, D. Christofilos, G. Bachelier, A. Crut, P. Maioli, N. Del Fatti, and F. Vallee, Phys. Rev. Lett. 107, 057402 (2011).

${ }^{30}$ M. Conforti and G. Della Valle, Phys. Rev. B 85, 245423 (2012). 